\title{
Development of Dictionary Entries for the Bangla Vowel Ended Roots for Universal Networking Language
}

\author{
Mohammad Zakir Hossain \\ Sarker \\ Department of Computer \\ Science and Engineering, \\ Jahangirnagar University \\ Dhaka, Bangladesh
}

\author{
Md. Nawab Yousuf Ali \\ Department of Computer \\ Science and Engineering, East \\ West University \\ Dhaka, Bangladesh
}

\author{
Jugal Krishna Das \\ Department of Computer \\ Science and Engineering, \\ Jahangirnagar University \\ Dhaka, Bangladesh
}

\begin{abstract}
Conversion from another language to native language is highly demanding due to increasing the usage of web based application. Firstly, the respective sentence of a native language is converted to Universal Networking Language (UNL) expressions and then UNL expressions can be converted to any native language. UNL system is developed for most of the languages already but a very little effort has been made to convert Bangla language to UNL expressions. In this paper, we have described Bangla verb, Verb root, Verbal Inflections and the finally developed the dictionary entries for the vowel ended roots and their respective verbal inflections.
\end{abstract}

\section{General Terms}

Universal Networking Language, Bangla Verb, Verb Root, Verbal Inflections

\section{Keywords}

Vowel Ended Group (VEG), Consonant Ended Group

\section{INTRODUCTION}

Bangla is the 4th widely spoken language with more than 250 million speakers, most of who live in Bangladesh and the Indian state of West Bengle [1]. Therefore it is essential to take into account a conversion process of Bangla to UNL and vice versa, so that a vast people can be benefited to share information in the Internet. The Universal Networking Language (UNL), which is a formal language for symbolizing the sense of natural language sentences, is a specification for the exchange of information. Currently, the UNL includes 16 languages [2], which are the six official languages of the United Nations (Arabic, Chinese, English, French, Russian and Spanish), in addition to the ten other widely spoken languages (German, Hindi, Italian, Indonesian, Japanese, Latvian, Mongol, Portuguese, Swahili and Thai). In the last few years, Machine Translation (MT) techniques have been applied to web environments. The growing amount of available multilingual information on the Internet and the Internet users has led to a justifiable interest on this area. Hundreds of millions of people of almost all levels of education, attitudes and different jobs all over the world use the Internet for different purposes [3], where English is the main language of the Internet. But English is not understandable for most of the people. Interlingua translation programs are needed to develop. The main goal of the UNL system, which allows users to visualize websites in their native languages, is to provide a common representation for accessing Internet of multilingual websites by the majority of Bangla or Bengali (বাংলা, pronounced as bangla) is an eastern Indo-Aryan language. It is native to the region of eastern South Asia known as Bengal, which comprises the people over the world. A significant part of the development of any machine translation (MT) system is the creation of lexical resources that the system will use. Dictionaries are of critical importance in MT. They are the largest components of an MT system in terms of the amount of information they hold. Generation of natural language from a machine processable, precise knowledge representation has to grapple with the problem of redundancy and impreciseness inherent in any natural language. In the UNL System [4], natural language analysis has been carried out by the EnConverter (EnCo) [5] associated with Word Dictionary of a native language and language specific analysis rules that converts a native language text into UNL expression and DeConverter (DeCo) [6] also associated with Word Dictionary that converts UNL expression to a variety of native languages using language specific generation rules. As our is to include Bangla in this system with less effort and we have been working for the last 4 years to achieve our goal. In this paper we have concentrated on Bangla Vowel Ended Roots and their related Verbal Inflections. Finally we have explained the lexicon (dictionary) entries of the Vowel Ended Roots.

The organization of this paper is as follow: In section 2 we describe the literatures that have been used to do this research work. Section 3 explains the Bangla verb, Verb root, verbal Inflections, etc., Section 4 shows the various Vowel Ended Group (VEG) that we have proposed for the system, Dictionary entries of the Vowel Ended Roots and Verbal Inflections are shown in section 5 \& 6. Finally, some concluding remarks are in Section 7.

\section{LITERATURE REVIEW}

For converting Bangla sentence to UNL expressions firstly, we have gone through Universal Networking Language (UNL) $[5,7,8]$ where we have learnt about UNL expression, Relations, Attributes, Universal Words, UNL Knowledge Base, Knowledge Representation in UNL, Logical Expression in UNL, UNL systems and specifications of Enconverter. All these are key factors for preparing Bangla word dictionary, dictionary entries, etc. Secondly, we have rigorously gone through the Bangla grammar $[9,10,11,16]$, Verb and roots (Vowel ended and Consonant Ended) [11, 12, 15], Primary suffixes [10, 12, 13, 14]. Using above references we extort ideas about Bangla grammar for morphological and semantic analysis in order to prepare Bangla word dictionary (for vowel ended root, root word suffix etc).

\section{ANALYSIS OF BANGLA VERB, VERB ROOT, VERB INFLECTIONS}

Bangladesh, the Indian state of West Bengal and parts of the Indian states of Tripura and Assam. Bangla is written in a script called the Bengali Script. Like other languages, the 
letters are grouped together based on the way they are pronounced. The first 11 letters are vowels followed by consonants.

\subsection{Bangla Verb ( क्रिয়া )}

Bangla verbs are highly inflected and are regular with few exceptions. They consist of a stem and an ending; they are traditionally listed in Bangla dictionaries in their "verbal noun" form, which is usually formed by adding - a (আ) to the stem, for instance, রাখা (rakha) means "to keep". The stem can end in either a vowel or a consonant, based on which Bangla verb roots can be divided into two categories, i.e. 1) Vowel Ended Root 2) Consonant Ended Root. As we have worked on Vowel Ended Roots, this paper will concentrate on Vowel Ended Roots more.

\subsection{Vowel Ended Root}

Bangla language has more than 30,000 verbs [12]. Verbs come from Roots and Verbal Inflections i.e. Verb = Verb Root + Verb Inflections. In Bangla there are around 25 Vowel Ended Roots. These are: भा(paa), था(kha), গा(gaa), চा(cha), ছা(chha), नि(ni), দি(di), या(ja), ছু(chhu), शু(thu), শু(shu),

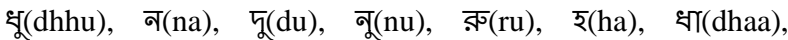
না(naa), বা (baa), ক(ka), ব(ba), র(ra) and স(sa)

\subsection{Verbal Inflections}

The inflections that are added after Verb Roots to make verbs are called Verbal Inflections [12]. For example, ই(i), ইতেছ্ছি(itechhi), এলাম(elam), etc.

\section{OUR PROPOSED CATEGORIZATION OF VOWEL ENDED ROOTS}

For appropriate morphological analysis and designing template of verb roots Vowel Ended Roots are categorized in 10 different categories based on the usages of Verbal Inflections. Categorizations are shown in the following tables. Here we have shown the tables for the first person only.

Table 1: Variations of Vowel Ended Roots and their verbal Inflections of VEG1, VEG1.1, VEG2 and VEG3 for $1^{\text {st }}$ Person

\begin{tabular}{|c|c|c|c|c|c|c|c|c|c|c|}
\hline \multicolumn{11}{|c|}{ Vowel Ended Roots } \\
\hline & & Tenses & भा & था & গा & छा & ছা & নি & দি & या \\
\hline & & Present Indefinite (Ch and Sh) & ই & ই & ই & ই & ই & ই & ই & ই \\
\hline & & Present Cont (Ch) & ष्टि & ष्্ि & ष्फि & ष्कि & फ्रि & ष्ছি & ष्टि & ष्্ि \\
\hline & & Present Cont (Sh) & ইতেছি & ইতেছ্ & ইতেছ্ & ইতেছি & ইতেছ্ & তেছ্ছি & তেছ্ছি & ইতেছ্ছি \\
\hline & 总 & Present Perfect (Ch) & $\begin{array}{l}\text { भा> } \\
\text { এয়েছি }\end{array}$ & $\begin{array}{l}\text { था>থ } \\
\text { এয়েছি }\end{array}$ & $\begin{array}{l}\text { গা>গ } \\
\text { এয়েছি }\end{array}$ & $\begin{array}{l}\text { চা>চ } \\
\text { এয়েছি }\end{array}$ & $\begin{array}{l}\text { ছা>ছ্ } \\
\text { এয়েছি }\end{array}$ & য়েছি & য়েছ্ & যা>গ ইয়েছি \\
\hline & & Present Perfect (Sh) & ইয়াছ্ & ইয়াছ্ & ইয়াছ্ ি & ইয়াছ্ & ইয়াছ্ & য়াছ্ & য়াছ্ & যা>গ ইয়াছ্ছি \\
\hline & & Imperative & $*$ & * & * & * & * & * & $*$ & * \\
\hline \multirow{10}{*}{ 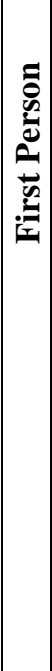 } & \multirow{7}{*}{$\vec{n}$} & Past Indef (Ch) & भा> & था>थ & ইলাম & ইলাম & ইলাম & লাম & লাম & যা>গ এলাম \\
\hline & & & এলাম & এলাম & & & & & & \\
\hline & & Past Indefinite (Sh) & ইলাম & ইলাম & ইলাম & ইলাম & ইলাম & লাম & লাম & यা>গ এলাম \\
\hline & & Past Continuous (Ch) & চ্ছিলাম & চ্ছিলাম & চ্ছিলাম & ष্ছিলাম & চ্ছিলাম & চ্ছিলাম & চ্ছিলাম & চ্ছিলাম \\
\hline & & Past Continuous (Sh) & $\begin{array}{l}\text { ইতেছ্ } \\
\text { লাম }\end{array}$ & $\begin{array}{l}\text { ইতেছ্ } \\
\text { লাম }\end{array}$ & $\begin{array}{l}\text { ইতেष্ছি } \\
\text { লाম }\end{array}$ & $\begin{array}{l}\text { ইতেष্ছিলা } \\
\text { म }\end{array}$ & $\begin{array}{l}\text { ইতেছ্ছিলা } \\
\text { म }\end{array}$ & তেছিলাম & তেছ্দিলাম & ইতেছিলাম \\
\hline & & Past Perfect (Ch) & $\begin{array}{l}\text { भा> } \\
\text { এয়েছি } \\
\text { লाম }\end{array}$ & $\begin{array}{l}\text { था>থ } \\
\text { এয়েছ্ } \\
\text { লाম }\end{array}$ & $\begin{array}{l}\text { গা>গ } \\
\text { এয়েছি } \\
\text { লাম }\end{array}$ & $\begin{array}{l}\text { চा>চ } \\
\text { এয়েছিলা } \\
\text { म }\end{array}$ & $\begin{array}{c}\text { ছা>ছ } \\
\text { এয়েছ্ছিলা } \\
\text { म }\end{array}$ & য়েছ্ছিলাম & য়েছ্ছিলাম & $\begin{array}{c}\text { यা>গ } \\
\text { ইয়েছ্ছিলাম }\end{array}$ \\
\hline & & Past Perfect (Sh) & $\begin{array}{l}\text { ইয়াছি } \\
\text { लाम }\end{array}$ & $\begin{array}{l}\text { ইয়াছি } \\
\text { লाম }\end{array}$ & $\begin{array}{l}\text { ইয়াছি } \\
\text { লाম }\end{array}$ & $\begin{array}{l}\text { ইয়াছ্ছিলা } \\
\text { म }\end{array}$ & $\begin{array}{l}\text { ইয়াছিলা } \\
\text { म }\end{array}$ & য়াছ্ছিলাম & য়াছ্ছিলাম & $\begin{array}{c}\text { या>গ } \\
\text { ইয়াছিলাম }\end{array}$ \\
\hline & \multirow{3}{*}{$\underset{\Xi}{\underline{\Xi}}$} & Future Indefinite (Ch) & বো, ব ব & বো, ব & বো, ব & বো, ব & বো, ব & বো, ব & বো, ব & বো, ব \\
\hline & & Future Indefinite(Sh) & $\begin{array}{l}\text { ইব, } \\
\text { ইবো }\end{array}$ & $\begin{array}{l}\text { ইব, } \\
\text { ইবো }\end{array}$ & $\begin{array}{l}\text { ইব, } \\
\text { ইবো }\end{array}$ & ইব, ইবো & ইব, ইবো & বো, ব & বো, ব & ইব, ইবো \\
\hline & & Imperative & $*$ & $*$ & $*$ & $*$ & $*$ & $*$ & $*$ & * \\
\hline & & & \multicolumn{2}{|c|}{ Group VEG1 } & \multicolumn{3}{|c|}{ Group VEG1.1 } & \multicolumn{2}{|c|}{ Group VEG2 } & Group VEG3 \\
\hline
\end{tabular}


Table 2: Variations of Vowel Ended Roots and their verbal Inflections of VEG4, VEG5, VEG6 and VEG7 for $1^{\text {st }}$ Person

\begin{tabular}{|c|c|c|c|c|c|c|c|c|c|c|c|}
\hline \multicolumn{12}{|c|}{ Vowel Ended Roots } \\
\hline & & Tenses & 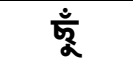 & থू & শ3 & $\nLeftarrow$ & न & দू & নू & রু & ल \\
\hline \multirow{15}{*}{ 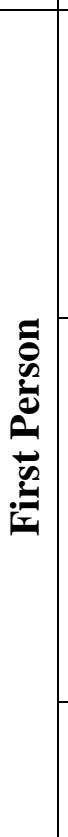 } & \multirow{6}{*}{ 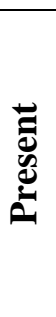 } & $\begin{array}{l}\text { Present Indefinite (Ch and } \\
\text { Sh) }\end{array}$ & ई & र & ই & ই & ই & ই & ই & ই & ই \\
\hline & & Present Cont $(\mathrm{Ch})$ & फ्रि & फ्कि & फ्रि & फ्कि & & फ्रि & फ्कि & फ्कि & फ्रि \\
\hline & & Present Cont (Sh) & ইতেছ্ & ইতেছি & ইতেছ্ & ইতেছ্ & & ইতেছ্ & ইতেছ্ & ইতেছি & ইতেছ্ \\
\hline & & Present Perfect $(\mathrm{Ch})$ & য়েছি & য়েছি & য়েছি & য়েছ্ & & য়েছি & য়েছি & য়েছি & য়েছ্ \\
\hline & & Present Perfect (Sh) & ইয়াছি & ইয়াছ্ & ইয়াছ্ & ইয়াছি & & ইয়াছি & ইয়াচ্ছি & ইয়াছি & ইয়াছ্ \\
\hline & & Imperative & $\star$ & * & * & * & & $*$ & $*$ & $*$ & $* *$ \\
\hline & \multirow{6}{*}{$\stackrel{\vec{n}}{E}$} & Past Indef (Ch) & लाম & লাম & লাম & লাম & & লাম & লাম & লাম & লাম \\
\hline & & Past Indefinite (Sh) & ইলাম & ইলাম & ইলাম & ইলাম & & ইলাম & ইলাম & ইলাম & ইলাম \\
\hline & & Past Continuous (Ch) & फ्रिलाম & फ्रिलाম & फ्रिलाম & फ्टिलाম & & फ्रिलाম & फ्रिलाম & फ्रिলাম & फ्टिলাম \\
\hline & & Past Continuous (Sh) & ইতেছিলাম & $\begin{array}{c}\text { ইতেष্ছিলা } \\
\text { म }\end{array}$ & ইতেছিলাম & ইতেছিলাম & & $\begin{array}{c}\text { ইতেছিলা } \\
\text { ম }\end{array}$ & ইতেছিলাম & ইতেছিলাম & ইতেছ্দিলাম \\
\hline & & Past Perfect (Ch) & য়েছিলাম & য়েছিলাম & য়েছিলাম & য়েছিলাম & & য়েছ্ছিলাম & য়েছিলাম & য়েছিলাম & য়েছ্ছিলাম \\
\hline & & Past Perfect (Sh) & ইয়াছিলাম & $\begin{array}{l}\text { ইয়াছিলা } \\
\text { ম }\end{array}$ & ইয়াছিলাম & ইয়াছিলাম & & $\begin{array}{l}\text { ইয়াছিলা } \\
\text { म }\end{array}$ & ইয়াছিলাম & ইয়াছিলাম & ইয়াছ্ছিলাম \\
\hline & \multirow{3}{*}{ 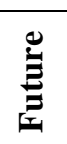 } & Future Indefinite(Ch) & ব & ব & ব & বো, ব & & বো, ব & বো, ব & বো, ব & বো, ব \\
\hline & & Future Indefinite(Sh) & ইব, ইবো & ইব, ইবো & ইব, ইবো & ইব, ইবো & & ইব, ইবো & ইব, ইবো & ইব, ইবো & ইব, ইবো \\
\hline & & Imperative & $\star$ & $\star$ & $\star$ & $*$ & $*$ & $*$ & $*$ & $*$ & $*$ \\
\hline & & & \multicolumn{4}{|c|}{ Gorup VEG4 } & $\begin{array}{l}\mathrm{VE} \\
\mathbf{G} 5\end{array}$ & \multicolumn{3}{|c|}{ Group VEG6 } & $\begin{array}{ll}\text { Group } \\
\text { VEG7 }\end{array}$ \\
\hline
\end{tabular}

Table 3: Variations of Vowel Ended Roots and their verbal Inflections of VEG8, VEG9 and VEG10 for $1^{\text {st }}$ Person

\begin{tabular}{|c|c|c|c|c|c|c|c|c|c|c|}
\hline \multicolumn{11}{|c|}{ Vowel Ended Roots } \\
\hline & & Tenses & ร & धा & ना & বा & ক & ব & 4 & ल \\
\hline \multirow{15}{*}{ 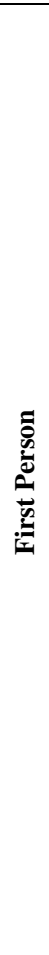 } & \multirow{6}{*}{ 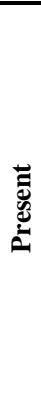 } & $\begin{array}{l}\text { Present Indefinite (Ch } \\
\text { and Sh) }\end{array}$ & रे & रे & रे & रे & $\bar{े}$ & ই & रे & ই \\
\hline & & Present Cont $(\mathrm{Ch})$ & फ्कि & फ्कि & फ्कि & फ्্ि & फ्ছি & फ्कि & फ्कि & फ्कि \\
\hline & & Present Cont (Sh) & ইতেছি & ইতেছি & ইতেছি & ইতেছ্ছি & ইতেচ্ছি & ইতেছ্ছি & ইতেছ্ছি & ইতেছ্ছি \\
\hline & & Present Perfect $(\mathrm{Ch})$ & য়েছ্ছি & $\begin{array}{l}\text { ধা>ধ } \\
\text { এয়েছি }\end{array}$ & $\begin{array}{l}\text { না>ন } \\
\text { এয়েছ্ }\end{array}$ & বা>ব এয়েছ্ছি & য়েছ্ & য়েছি & য়েছি & য়েছি \\
\hline & & Present Perfect (Sh) & ইয়াছ্ & ইয়াছ্ছি & ইয়াছি & ইয়াছি & ইয়াছি & ইয়াছি & ইয়াছি & ইয়াছি \\
\hline & & Imperative & $\star$ & * & $\star$ & $\star$ & $*$ & $*$ & $*$ & $*$ \\
\hline & \multirow{6}{*}{$\stackrel{\vec{a}}{\vec{\theta}}$} & Past Indef (Ch) & লাম & ইলাম & ইলাম & ইলাম & লাম & লাম & লাম & লাম \\
\hline & & Past Indefinite (Sh) & ইলাম & ইলাম & ইলাম & ইলাম & ইলাম & ইলাম & ইলাম & ইলাম \\
\hline & & Past Continuous (Ch) & ष্ছিলাম & চ্ছিলাম & फ্्িলাম & চ্ছিলাম & চ্ছিলাম & চ্ছিলাম & চ্ছিলাম & চ্ছিলাম \\
\hline & & Past Continuous (Sh) & ইতেছ্ছিলাম & ইতেচ্ছিলাম & ইতেচ্ছিলাম & ইতেছ্ছিলাম & ইতেছ্ছিলাম & ইতেছ্ছিলাম & ইতেচ্ছিলাম & ইতেচ্ছিলাম \\
\hline & & Past Perfect (Ch) & য়েছিলাম & $\begin{array}{c}\text { ধা>ধ } \\
\text { এয়েছ্ছিলাম }\end{array}$ & $\begin{array}{c}\text { না>ন } \\
\text { এয়েছ্ছিলাম }\end{array}$ & $\begin{array}{c}\text { বা>ব } \\
\text { এয়েছিলাম }\end{array}$ & য়েছিলাম & য়েছিলাম & য়েছিলাম & য়েছ্ছিলাম \\
\hline & & Past Perfect (Sh) & ইয়াছিলাম & ইয়াছিলাম & ইয়াছিলাম & ইয়াছিলাম & ইয়াছিলাম & ইয়াছিলাম & ইয়াছিলাম & ইয়াছ্ছিলাম \\
\hline & \multirow{3}{*}{$\underset{\Xi}{\Xi}$} & Future Indefinite(Ch) & ব & ব & ব & ব & বো, ব & বো, ব & বো, ব & বো, ব \\
\hline & & Future Indefinite(Sh) & ইব, ইবো & ইব, ইবো & ইব, ইবো & ইব, ইবো & ইব, ইবো & ইব, ইবো & ইব, ইবো & ইব, ইবো \\
\hline & & Imperative & * & * & * & $\star$ & $*$ & $*$ & $*$ & $*$ \\
\hline & & & VEG8 & \multicolumn{3}{|c|}{ Group VEG9 } & \multicolumn{4}{|c|}{ Group VEG10 } \\
\hline
\end{tabular}




\section{OUR PROPOSED DICTIONARY ENTRIES OF VOWEL ENDED ROOTS}

Attribute's Descriptions:

VR-Verb Root,

VE-Vowel Ended, DEF-Default,

VEG-Vowel Ended Group

\section{Group VEG1}

[भा] \{\}“...”(VR,VE,DEF,VEG1)

[भ] \{\}“...”(VR,VE, ALT,VEG1)

[था] \{\}“...”(VR,VE,DEF,VEG1)

$[थ]\{\} “ . . . ”(V R, V E$, ALT,VEG1)

[গा] \{\}“...”(VR,VE,DEF,VEG1.1)

[গ] \{\}“...”(VR,VE, ALT,VEG1.1)

[চा] \{\}“...”(VR,VE,DEF,VEG1.1)

[চ] \{\}“...” (VR,VE, ALT,VEG1.1)

[ছা] \{\}“...”(VR,VE,DEF,VEG1.1)

[ছ] \{\}“...”(VR,VE, ALT,VEG1.1)

\section{Group VEG2}

[नि] \{\}“...”(VR,VE,DEF,VEG2)

[ब] \{\}“...” (VR,VE, ALT,VEG2)

[দি] \{\}“...” (VR,VE,DEF,VEG1)

[ฤ] \{\}“...”(VR,VE, ALT,VEG2)

\section{Group VEG3}

[या] \{\}“...” (VR,VE,DEF,VEG3)

[গ] \{\}“...” (VR,VE, ALT,VEG3)

\section{Group VEG4}

[ฆूँ] \{\}“...” (VR,VE,DEF,VEG4)

[ছো] \{\}“...” (VR,VE,ALT,VEG4)

[शू \{\}“...” (VR,VE,DEF,VEG4)

[থো] \{\}“...” (VR,VE,ALT,VEG4)

[শु] \{\}“...” (VR,VE,DEF,VEG4)

[শো] \{\}“...” (VR,VE,ALT,VEG4)

[ধ్ম \{\}“...” (VR,VE,DEF,VEG4)

[ধধা] \{\}“...” (VR,VE,ALT,VEG4)

\section{Group VEG5}

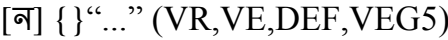

\section{Group VEG6}

[7] \{\}“...” (VR,VE,DEF,VEG6) [দো] \{\}“...” (VR,VE,ALT,VEG6)

[নু \{\}“...”(VR,VE,DEF,VEG6)
[নো] \{\}“...” (VR,VE,ALT,VEG6)

[রু] \{\}“...” (VR,VE,DEF,VEG6)

[রো] \{\}“....”(VR,VE, ALT,VEG6)

\section{Group VEG7}

[ल] \{\}$“ . . . ”(V R, V E, D E F, V E G 7)$

\section{Group VEG8}

[₹] \{\}“...”(VR,VE,DEF,VEG8)

\section{Group VEG9}

[ধा] \{\}“...”(VR,VE,DEF,VEG9)

[ধ] \{\}“...”(VR,VE,ALT,VEG9)

[ना] \{\}$“ . . . ”(V R, V E, D E F, V E G 9)$

[ब] \{\}“...”(VR,VE,ALT,VEG9)

[বा] \{\}$“ . . . ”(V R, V E, D E F, V E G 9)$

[ব] \{\}$“ . . . ”(V R, V E, A L T, V E G 9)$

\section{Group VEG10}

[क] \{\}$“ . . . ”(V R, V E, D E F, V E G 10)$

[ব] \{\}“...”(VR,VE,DEF,VEG10)

[ส] \{\}“...”(VR,VE,DEF,VEG10)

[স] \{\}“...”(VR,VE,DEF,VEG10)

\section{DICTIONARY ENTRIES OF VERBAL INFLEXIONS}

\section{First Person}

Present Tense

[ই] “” \{\}$(\mathrm{VI}, \mathrm{P} 1, \mathrm{PRT}, \mathrm{DEF}){ }^{\circledR}$

[ष्कि] “ " \{\}$(\mathrm{VI}, \mathrm{P} 1, \mathrm{PRT}, \mathrm{PRG}, \mathrm{DEF}, \mathrm{CH}){ }^{\circledR}$

[ইতেছি “ “” \{\}$(\mathrm{VI}, \mathrm{P} 1, \mathrm{PRT}, \mathrm{PRG}, \mathrm{DEF}, \mathrm{SD}, \wedge \mathrm{VEG} 2){ }^{\circledR}$

[তেছ্ছি “ ” $\left\{(\mathrm{VI}, \mathrm{P} 1, \mathrm{PRT}, \mathrm{PRG}, \mathrm{DEF}, \mathrm{SD}, \mathrm{VEG} 2){ }^{\circledR}\right.$

[এয়েছি] “ ”\{\}(VI,P1,PRT, PER, ALT,CH, VEG1, VEG1.1,

VEG9)®

[য়েছি] “" "\{\}(VI,P1,PRT, PER, DEF,CH, VEG2, VEG4,

VEG6,VEG7, VEG8, VEG10) ®

[ইग़েছি] “ " \{\}$(\mathrm{VI}, \mathrm{P} 1, \mathrm{PRT}, \mathrm{PER}, \mathrm{ALT}, \mathrm{CH}, \mathrm{VEG} 3)$ ®

[ইয়াছি “ “” $\left\{\left(\mathrm{VI}, \mathrm{P} 1, \mathrm{PRT}, \mathrm{PER}, \mathrm{DEF}, \mathrm{SD}, \wedge \mathrm{VEG} 2,{ }^{\wedge} \mathrm{VEG} 3\right){ }^{\circledR}\right.$

[ইয়াছি] “" \{\}(VI,P1,PRT, PER, ALT,SD,VEG3) ®

Past Tense

[এলাম] “ ” \{\}$\left(\mathrm{VI}, \mathrm{P} 1, \mathrm{PST}, \mathrm{ALT}, \mathrm{CH}, \mathrm{VEG1,VEG3)}{ }^{\circledR}\right.$ [ইলাম] “ ” \{\}$(V I, P 1, P S T, D E F, C H, V E G 1.1, V E G 9) \circledR$ [लाম] “" \{\}(VI,P1,PST,DEF,CH,VEG2,VEG4, VEG6,VEG7, VEG8, VEG10) ${ }^{\circledR}$

[ইলাম] “ ” \{\}$\left(\mathrm{VI}, \mathrm{P} 1, \mathrm{PST}, \mathrm{DEF}, \mathrm{SD},{ }^{\wedge} \mathrm{VEG} 2,{ }^{\wedge} \mathrm{VEG} 3\right) \AA$ 


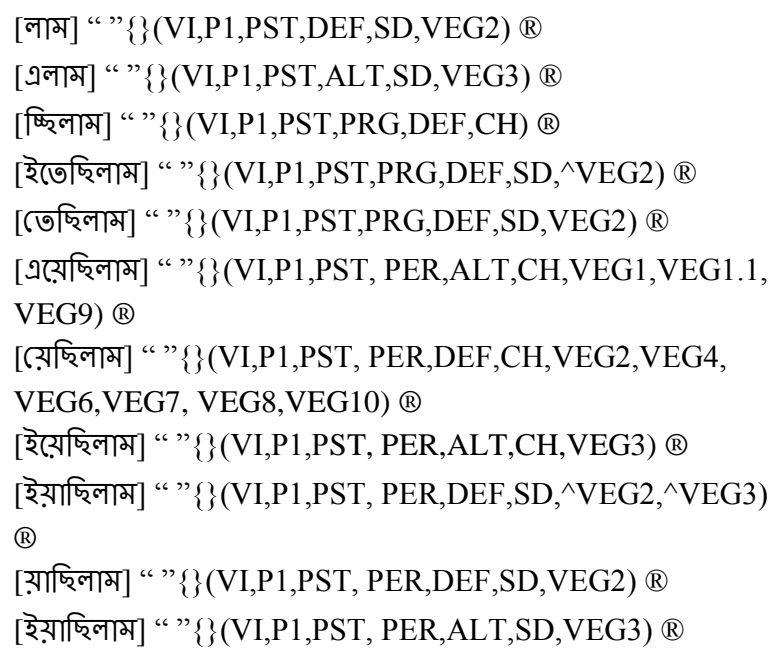

\section{Future Tense}

[বো] “ ” \{\}$(\mathrm{VI}, \mathrm{P} 1, \mathrm{FUT}, \mathrm{DEF}, \mathrm{CH}){ }^{\circledR}$

[ব] “ ” \{\}$(\mathrm{VI}, \mathrm{P} 1, \mathrm{FUT}, \mathrm{DEF}, \mathrm{CH}){ }^{\circledR}$

[ইব] “ ” \{\}$\left(\mathrm{VI}, \mathrm{P} 1, \mathrm{FUT}, \mathrm{DEF}, \mathrm{SD},{ }^{\wedge} \mathrm{VEG} 2\right){ }^{\circledR}$

[ইবো] “ ” \{\}$(\mathrm{VI}, \mathrm{P} 1, \mathrm{FUT}, \mathrm{DEF}, \mathrm{SD}, \wedge \mathrm{VEG} 2){ }^{\circledR}$

[বো] “ ” \{\}$(\mathrm{VI}, \mathrm{P} 1, \mathrm{FUT}, \mathrm{DEF}, \mathrm{SD}){ }^{\circledR}$

[ব] “ ” \{\}$(\mathrm{VI}, \mathrm{P} 1, \mathrm{FUT}, \mathrm{DEF}, \mathrm{SD}, \mathrm{VEG} 2){ }^{\circledR}$

\section{$\underline{\text { Second Person }}$}

\section{Present Tense}

[3] “ ” \{\}$(\mathrm{VI}, \mathrm{P} 2, \mathrm{GEN}, \mathrm{PRT}, \mathrm{DEF}){ }^{\circledR}$

[স] “" \{\}$\left(\right.$ VI,P2,NEG,PRT,DEF) ${ }^{\circledR}$

[न] “ ” \{\}$(\mathrm{VI}, \mathrm{P} 2, \mathrm{RES}, \mathrm{PRT}, \mathrm{DEF}) \circledR$

[ष्ছ] “ ” \{\}$(\mathrm{VI}, \mathrm{P} 2, \mathrm{GEN}, \mathrm{PRT}, \mathrm{PRG}, \mathrm{DEF}, \mathrm{CH}){ }^{\circledR}$

[ष्ছिস] “ " \{\}$(\mathrm{VI}, \mathrm{P} 2, \mathrm{NEG}, \mathrm{PRT}, \mathrm{PRG}, \mathrm{DEF}, \mathrm{CH}){ }^{\circledR}$ [ष्ছেন] “ ” \{\}$($ VI,P2,RES,PRT, PRG,DEF,CH) ® [ইতেছ্] “ ” \{\}$($ VI,P2,GEN,PRT,PRG,DEF,SD) ® [ইতেছ্ছিস] “ ” \{\}$(\mathrm{VI}, \mathrm{P} 2, \mathrm{NEG}, \mathrm{PRT}, \mathrm{PRG}, \mathrm{DEF}, \mathrm{SD}){ }^{\circledR}$ [ইতেছ্নেন] “ ” \{\}$(\mathrm{VI}, \mathrm{P} 2, \mathrm{RES}, \mathrm{PRT}, \mathrm{PRG}, \mathrm{DEF}, \mathrm{SD}){ }^{\circledR}$ [এয়েছ্] “ ” \{\}$(\mathrm{VI}, \mathrm{P} 2, \mathrm{GEN}, \mathrm{PRT}, \mathrm{PER}, \mathrm{ALT}, \mathrm{CH}){ }^{\circledR}$

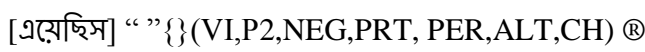
[এয়েছ্নেন] “ " \{\}$(\mathrm{VI}, \mathrm{P} 2, \mathrm{RES}, \mathrm{PRT}, \mathrm{PER}, \mathrm{ALT}, \mathrm{CH}){ }^{\circledR}$ [ইয়াছ] “ " \{\}$(\mathrm{VI}, \mathrm{P} 2, \mathrm{GEN}, \mathrm{PRT}, \mathrm{PER}, \mathrm{DEF}, \mathrm{SD}){ }^{\circledR}$ [ইয়াছ্ছিস] “ ” \{\}$(\mathrm{VI}, \mathrm{P} 2, \mathrm{NEG}, \mathrm{PRT}, \mathrm{PER}, \mathrm{DEF}, \mathrm{SD}){ }^{\circledR}$ [ইয়াছ্নেন] “ ” \{\}$(\mathrm{VI}, \mathrm{P} 2, \mathrm{RES}, \mathrm{PRT}, \mathrm{PER}, \mathrm{DEF}, \mathrm{SD})$ (®)

\section{Past Tense}

[এলে] “ ” \{\}$(\mathrm{VI}, \mathrm{P} 2, \mathrm{GEN}, \mathrm{PST}, \mathrm{ALT}, \mathrm{CH}){ }^{\circledR}$ [এলি] “ ” \{\}$(\mathrm{VI}, \mathrm{P} 2, \mathrm{NEG}, \mathrm{PST}, \mathrm{ALT}, \mathrm{CH}){ }^{\circledR}$ [এলেন] “ ” \{\}$(\mathrm{VI}, \mathrm{P} 2, \mathrm{RES}, \mathrm{PST}, \mathrm{ALT}, \mathrm{CH}){ }^{\circledR}$ [ইলে] “ ” \{\}$(\mathrm{VI}, \mathrm{P} 2, \mathrm{GEN}, \mathrm{PST}, \mathrm{DEF}, \mathrm{SD}){ }^{\circledR}$ [ইলি] “ ” \{\}$(\mathrm{VI}, \mathrm{P} 2, \mathrm{NEG}, \mathrm{PST}, \mathrm{DEF}, \mathrm{SD}) \circledR$ [ইলেন] “ ” \{\}$(\mathrm{VI}, \mathrm{P} 2, \mathrm{RES}, \mathrm{PST}, \mathrm{DEF}, \mathrm{SD}){ }^{\circledR}$
[এতে] “ ” \{\}$(\mathrm{VI}, \mathrm{P} 2, \mathrm{GEN}, \mathrm{PST}, \mathrm{ALT}, \mathrm{CH}){ }^{\circledR}$ [এতিস] “ ” \{\}$(\mathrm{VI}, \mathrm{P} 2, \mathrm{NEG}, \mathrm{PST}, \mathrm{ALT}, \mathrm{CH}){ }^{\circledR}$ [এতেন] “ ”, $\}$ (VI,P2,RES,PST, ALT,CH) ® [ইতে] “ " \{\}$($ VI,P2,GEN,PST,DEF,SD) ® [ইতিস] “ ” \{\}$(\mathrm{VI}, \mathrm{P} 2, \mathrm{NEG}, \mathrm{PST}, \mathrm{DEF}, \mathrm{SD}){ }^{\circledR}$ [ইতেন] “ " \{\}$(\mathrm{VI}, \mathrm{P} 2, \mathrm{RES}, \mathrm{PST}, \mathrm{DEF}, \mathrm{SD})$ ( [ष্ছিলে] “ ” \{\}$(\mathrm{VI}, \mathrm{P} 2, \mathrm{GEN}, \mathrm{PST}, \mathrm{PRG}, \mathrm{DEF}, \mathrm{CH}){ }^{\circledR}$ [ष्ছিলি] “ " \{\}$(\mathrm{VI}, \mathrm{P} 2, \mathrm{GEN}, \mathrm{PST}, \mathrm{PRG}, \mathrm{DEF}, \mathrm{CH}){ }^{\circledR}$ [চ্ছিলেন] “ " \{\}$(\mathrm{VI}, \mathrm{P} 2, \mathrm{GEN}, \mathrm{PST}, \mathrm{PRG}, \mathrm{DEF}, \mathrm{CH}){ }^{\circledR}$

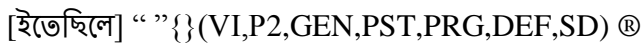

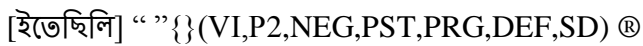
[ইতেছ্ছিলেন] “ " \{\}$($ VI,P2,RES,PST,PRG,DEF,SD) ® [এয়েছ্ছিলে] “ " \{\}$(\mathrm{VI}, \mathrm{P} 2, \mathrm{GEN}, \mathrm{PST}, \mathrm{PER}, \mathrm{ALT}, \mathrm{CH}){ }^{\circledR}$ [এয়েছ্ছিলি] “ " \{\}$(\mathrm{VI}, \mathrm{P} 2, \mathrm{NEG}, \mathrm{PST}, \mathrm{PER}, \mathrm{ALT}, \mathrm{CH}){ }^{\circledR}$ [এয়েছিলেন] “ " \{\}$(\mathrm{VI}, \mathrm{P} 2, \mathrm{RES}, \mathrm{PST}, \mathrm{PER}, \mathrm{ALT}, \mathrm{CH}){ }^{\circledR}$ [ইয়াছিলে] “ " " \{\}$(\mathrm{VI}, \mathrm{P} 2, \mathrm{GEN}, \mathrm{PST}, \mathrm{PER}, \mathrm{DEF}, \mathrm{SD}){ }^{\circledR}$ [ইয়াছিলি] “ ” \{\}$(\mathrm{VI}, \mathrm{P} 2, \mathrm{NEG}, \mathrm{PST}, \mathrm{PER}, \mathrm{DEF}, \mathrm{SD}){ }^{\circledR}$ [ইয়াছিলেন] “ ” \{\}$(\mathrm{VI}, \mathrm{P} 2, \mathrm{RES}, \mathrm{PST}, \mathrm{PER}, \mathrm{DEF}, \mathrm{SD}){ }^{\circledR}$

\section{Future Tense}

[বে] “ ” \{\}$(\mathrm{VI}, \mathrm{P} 2, \mathrm{GEN}, \mathrm{FUT}, \mathrm{DEF}, \mathrm{CH}){ }^{\circledR}$ [বি] “ ” \{\}$(\mathrm{VI}, \mathrm{P} 2, \mathrm{NEG}, \mathrm{FUT}, \mathrm{DEF}, \mathrm{CH}){ }^{\circledR}$ [বেন] “ " \{\}$(\mathrm{VI}, \mathrm{P} 2, \mathrm{RES}, \mathrm{FUT}, \mathrm{DEF}, \mathrm{CH}){ }^{\circledR}$ [ইবে] “ ” \{\}$(\mathrm{VI}, \mathrm{P} 2, \mathrm{GEN}, \mathrm{FUT}, \mathrm{DEF}, \mathrm{SD}){ }^{\circledR}$ [ইবি] “ " \{\}$(\mathrm{VI}, \mathrm{P} 2, \mathrm{GEN}, \mathrm{FUT}, \mathrm{DEF}, \mathrm{SD})$ ( [ইবেন] “ ” \{\}$(\mathrm{VI}, \mathrm{P} 2, \mathrm{GEN}, \mathrm{FUT}, \mathrm{DEF}, \mathrm{SD})$ ® [এও] “ ” \{\}$(\mathrm{VI}, \mathrm{P} 2, \mathrm{GEN}, \mathrm{FUT}, \mathrm{DEF}, \mathrm{CH})$ ® [ইস] “ ” \{\}$(\mathrm{VI}, \mathrm{P} 2, \mathrm{NEG}, \mathrm{FUT}, \mathrm{DEF}, \mathrm{CH}) \AA$ [ইও] “ ” \{\}$(\mathrm{VI}, \mathrm{P} 2, \mathrm{GEN}, \mathrm{FUT}, \mathrm{DEF}, \mathrm{SD}){ }^{\circledR}$ [স] “ ” \{\}$(\mathrm{VI}, \mathrm{P} 2, \mathrm{NEG}, \mathrm{FUT}, \mathrm{DEF}, \mathrm{SD}){ }^{\circledR}$

\section{Third Person}

\section{Present Tense}

[?]] “ ” \{\}$($ VI,P3,GEN,PRT,DEF) $®$

[न] “ ” \{\}$(\mathrm{VI}, \mathrm{P} 3, \mathrm{RES}, \mathrm{PRT}, \mathrm{DEF}){ }^{\circledR}$

[এয়] “ ” \{\}$(\mathrm{VI}, \mathrm{P} 3, \mathrm{GEN}, \mathrm{PRT}, \mathrm{ALT}){ }^{\circledR}$

[এন] “ ” \{\}$(\mathrm{VI}, \mathrm{P3}, \mathrm{GEN}, \mathrm{PRT}, \mathrm{ALT}){ }^{\circledR}$

[ष्্口] “ ” \{\}$($ VI,P3,GEN,PRT,PRG,DEF,CH) ® [চ্ছেন] “ " \{\}$(\mathrm{VI}, \mathrm{P} 3, \mathrm{RES}, \mathrm{PRT}, \mathrm{PRG}, \mathrm{DEF}, \mathrm{CH}){ }^{\circledR}$ [ইতেছে] “ ” \{\}$(\mathrm{VI}, \mathrm{P} 3, \mathrm{GEN}, \mathrm{PRT}, \mathrm{PRG}, \mathrm{DEF}, \mathrm{SD})$ ® [ইতেছ্নেন] “ " \{\}$(\mathrm{VI}, \mathrm{P} 3, \mathrm{RES}, \mathrm{PRT}, \mathrm{PRG}, \mathrm{DEF}, \mathrm{SD}){ }^{\circledR}$ [এয়েছে] “ " " \{\}$(\mathrm{VI}, \mathrm{P} 3, \mathrm{GEN}, \mathrm{PRT}, \mathrm{PER}, \mathrm{ALT}, \mathrm{CH}){ }^{\circledR}$ [এয়েছ্নেন] “ " \{\}$(\mathrm{VI}, \mathrm{P} 3, \mathrm{RES}, \mathrm{PRT}, \mathrm{PER}, \mathrm{ALT}, \mathrm{CH}){ }^{\circledR}$ [ইয়াছে] “ ” \{\}$(V I, P 3, G E N, P R T, P E R, D E F, S D) ~ \circledR$ [ইয়াছ্নেন] “" \{\}$(\mathrm{VI}, \mathrm{P} 3, \mathrm{RES}, \mathrm{PRT}, \mathrm{PER}, \mathrm{DEF}, \mathrm{SD}){ }^{\circledR}$

\section{Past Tense}

[এল] “ ”\{ $\}(\mathrm{VI}, \mathrm{P} 3, \mathrm{GEN}, \mathrm{PST}, \mathrm{ALT}, \mathrm{CH}){ }^{\circledR}$ 


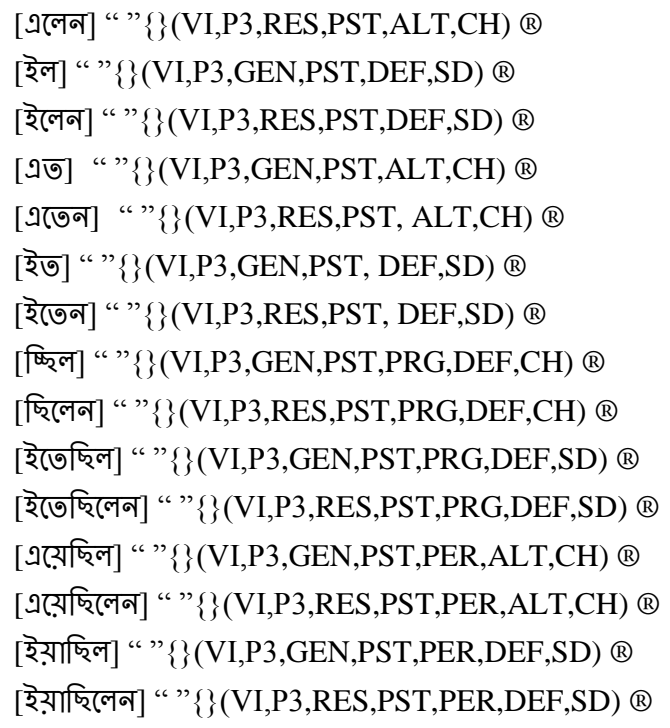

\section{Future Tense}

[বে] “ ” \{\}$\left(\right.$ VI,P3,GEN,FUT,DEF,CH) ${ }^{\circledR}$

[বেন] “ " \{\}$(\mathrm{VI}, \mathrm{P} 3, \mathrm{RES}, \mathrm{FUT}, \mathrm{DEF}, \mathrm{CH}){ }^{\circledR}$

[ইবে] “ ” \{\}$(\mathrm{VI}, \mathrm{P} 3, \mathrm{GEN}, \mathrm{FUT}, \mathrm{DEF}, \mathrm{SD}){ }^{\circledR}$

[ইবেন] “ ” \{\}$(\mathrm{VI}, \mathrm{P} 3, \mathrm{RES}, \mathrm{FUT}, \mathrm{DEF}, \mathrm{SD}) \mathbb{}$

\section{CONCLUSION AND FUTURE WORKS}

We have shown dictionary entries for Bangla Vowel Ended Roots and their respective verbal Inflections that are useful for conversion of Bangla sentences to UNL expression. In future we'll work on the Consonant Ended Roots and develop the dictionary entries in the same manner. Then we'll use the enconvertor to see the result.

\section{REFERENCES}

[1] D. M. Shohidullah, "Bangala Bayakaron", Ahmed Mahmudul Haque of Mowla Brothers Prokashoni, Dhaka, 2003

[2] Md. Ershadul H. Choudhury, Md. Nawab Yousuf Ali, Mohammad Zakir Hossain Sarker, Ahsan Razib, "Bridging Bangla to Universal Networking Language- A Human Language Neutral Meta-Language", International Conference on Computer, and Information Technology (ICCIT), Dhaka, 2005

[3] S. Abdel-Rahim, A.A. Libdeh, F. Sawalha, M. K. Odeh, "Universal Networking Language(UNL) a Means to Bridge the Digital Divide", Computer Technology Training and Industrial Studies Center, Royal Scientific Society, March 2002
[4] Uchida H., Zhu M., and Senta T. C. D., Universal Networking Language, UNDL Foundation, International environment house, 2005/6, Geneva, Switzerland

[5] EnConverter Specification, Version 3.3, UNL Center/UNDL Foundation, Tokyo 150-8304, Japan 2002

[6] DeConverter Specification, Version 2.7, UNL Center, UNDL Foundation, Tokyo 150-8304, Japan 2002

[7] Uchida H., Zhu M., “ The Universal Networking Language (UNL) Specification Version 3.0 1998, Technical Report, UNU, Tokyo, 1998”, 2005/6-UNDL Foundation, International Environment House

[8] Enconverter Specification Version 3.3, UNU Centre, Tokyo 150-8304, Japan 2002

[9] D.M. Shahidullah, "Bangla Baykaron", Ahmed Mahmudul Haque of Mowla Brothers prokashani, Dhaka-2003.

[10] D. C. Shuniti Kumar, "Bhasha-Prakash Bangala Vyakaran", Rupa and Company Prokashoni, Calcutta, July 1999, pp.170-175

[11] Asad H., "Bakkotottyo", Second edition, 1994, Dhaka

[12] D. S. Rameswar, "Shadharan Vasha Biggan and Bangla Vasha”, Pustok Biponi Prokashoni, November 1996, pp.358-377

[13] Md. Nawab Yousuf Ali, Mohammad Zakir Hossain Sarker , Ghulam Farooque Ahmed, Jugal Krishna Das."Conversion of Bangla Sentence into Universal Networking Language Expression", International Journal of Computer Science Issues, Vol. 8, Issue 2, March 2011, pp. 64-73

[14] M.N.Y. Ali, J.K. Das, S. M. Abdullah Al Mamun, M. E. H. Choudhury. "Specific Features of a Converter of Web Documents from Bengali to Universal Networking Language", International Conference on Computer and Communication Engineering 2008 (ICCCE'08), Kuala Lumpur, Malaysia. pp. 726-731

[15] M.N.Y. Ali, J.K. Das, S.M. Abdullah Al Mamun, A. M. Nurannabi. "Morphological Analysis of Bangla words for Universal Networking Language", International Conference on Digital Information Management, icdim, 2008, London, England, pp. 532-537

[16] M.N.Y.Ali, A. M. Nurannabi, G. F. Ahmed, J.K. Das. "Conversion of Bangla Sentence for Universal Networking Language", International Conference on Computer and Information Technology (ICCIT), Dhaka, 2010 pp. $108-113$ 